\title{
Spectrum of Lung and Cardiovascular Diseases in Association with Pulmonary Interstitial Glycogenosis \\ Rose Chami*
}

Division of Pathology, Department of Paediatric Laboratory Medicine, The Hospital for Sick Children and University of Toronto, Toronto, Ontario, Canada

\section{Article Info}

\section{Article Notes}

Received: October 24, 2018

Accepted: December 17, 2018

\section{*Correspondence:}

Dr. Rose Chami, Division of Pathology, Department of Paediatric Laboratory Medicine, The Hospital for Sick

Children and University of Toronto, Toronto, Ontario, Canada; Email: rose.chami@sickkids.ca

( 2018 Chami R. This article is distributed under the terms of the Creative Commons Attribution 4.0 International License.

\section{Keywords}

Infants

Pulmonary interstitial glycogenosis

Congenital pulmonary airway malformation

Growth alveolar abnormality

Hyperplasia of pulmonary neuroendocrine cells

Mesenchymal cells

\section{Abstract}

"Pulmonary Interstitial Glycogenosis (PIG) associated with a spectrum of neonatal pulmonary disorders", reported by Cutz et al represents one of the largest series published to date. The report included twenty-eight cases of lung or cardiac disorders with coincident diffuse, patchy, or focal PIG reviewed in Division of Pathology, The Hospital for Sick Children. The authors focused on reporting a spectrum of disorders associated with PIG and described four clinicopathological subgroups including imaging, ultrastructural findings, and clinical outcome. The present paper highlights the main findings reported by Cutz et al, and a review of literature is also presented.

PIG is a rare interstitial lung disease of infancy that is placed in the category of "specific conditions of undefined etiology" in the childhood interstitial lung disease (ChILD) classification (1). PIG has been reported mainly in either pre-term or full-term babies younger than six months of age $(2,3)$. Infants with PIG usually present with persistent tachypnea and hypoxemia shortly after birth or in the first few weeks of life $(3,4,5)$. The reported PIG cases have been described in otherwise normal lungs (isolated/diffuse or patchy pattern)

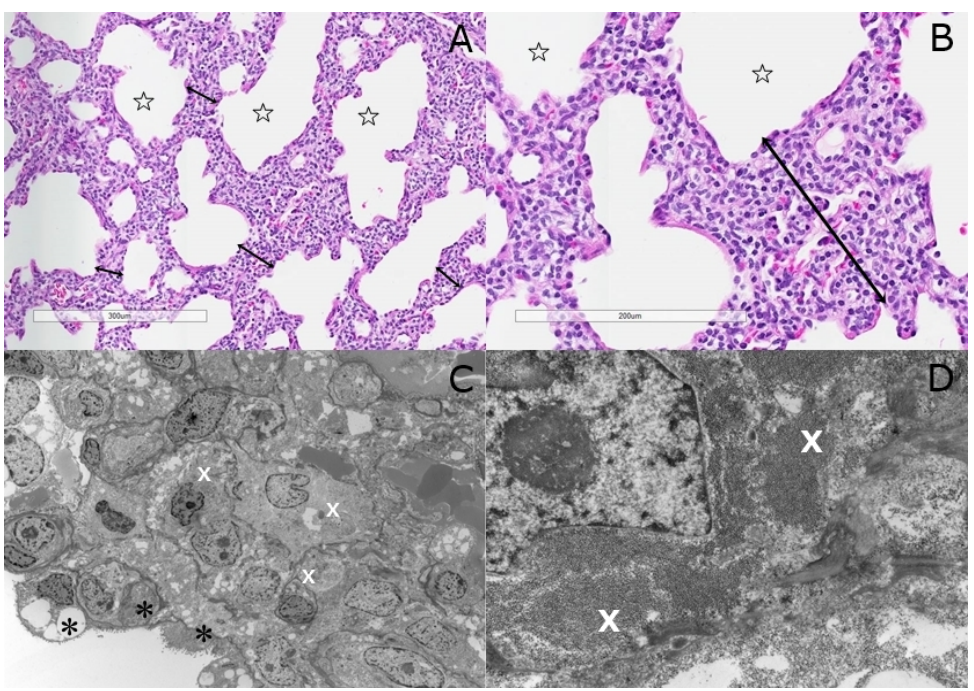

Figure 1: (A) Abnormal enlarged alveoli (stars) with diffuse interstitia thickening (double head arrows) with mesenchymal cells [H\&E stain]; (B) Interstitial mesenchymal cells with pale cytoplasm (H\&E stain); (C) Mesenchymal cells with rare organelles and abundant monoparticulate glycogen in cytoplasm (white X) and pneumocytes type II (black Asterix) [Transmission EM]; (D) Close-up view of PIG cells with pools of glycogen (white $\mathrm{X}$ ) [Transmission EM]. 
$(3,5)$ or often in conjunction with other cardiovascular or pulmonary abnormalities $(2,3,6,7)$. To the best of my knowledge, approximately 78 cases of patients with PIG (not including the cases published by Cutz et al) have been reported in the literature with variable information regarding the clinical presentation, comorbidities, imaging, histopathology, and outcome were published. The described findings are summarized in Table 1.

\begin{tabular}{|c|c|c|c|c|c|c|c|c|c|c|}
\hline Authors & $\begin{array}{l}\text { N. of } \\
\text { patients }\end{array}$ & $\begin{array}{l}\text { GA at } \\
\text { birth, } \\
\text { preterm or } \\
\text { term }\end{array}$ & Sex & $\begin{array}{l}\text { Age at } \\
\text { presentation }\end{array}$ & CHD & PHN & $\begin{array}{l}\text { Other } \\
\text { comorbidities }\end{array}$ & $\begin{array}{l}\text { Lung histopathology } \\
\text { (main findings) }\end{array}$ & $\begin{array}{l}\text { Chest HRCT } \\
\text { (main } \\
\text { findings) }\end{array}$ & $\begin{array}{l}\text { Outcome at time } \\
\text { of last follow-up }\end{array}$ \\
\hline $\begin{array}{l}\text { Liptzin et al, } \\
2018(6)\end{array}$ & 24 & $\begin{array}{l}\text { Late } \\
\text { preterm/ } \\
\text { term } \\
(\mathrm{N}=18)\end{array}$ & $\begin{array}{l}M \\
(\mathrm{~N}=15) \\
F(N=9)\end{array}$ & $\begin{array}{l}\text { Range 0.3-6 } \\
\text { months }\end{array}$ & $\begin{array}{l}63 \% \text { of patients } \\
\text { [including } \\
\text { HLHS, PVS, ASD, } \\
\text { VSD], PDA, TF, } \\
\text { mitral stenosis, } \\
\text { coarctation of } \\
\text { aorta) }\end{array}$ & $\begin{array}{l}38 \% \text { of } \\
\text { patients } \\
(\mathrm{N}=9)\end{array}$ & $\begin{array}{l}\text { Cleft lip/palate } \\
(\mathrm{N}=2) \\
\text { Autism ( } \mathrm{N}=1) \\
\text { Unilateral kidney } \\
(\mathrm{N}=1) \\
\text { Neurologic deficits } \\
(\mathrm{N}=4) \\
\text { Hypotonia }(\mathrm{N}=1) \\
\text { Seizure }(\mathrm{N}=1) \\
\text { Hypothyroidism } \\
(\mathrm{N}=1) \\
\text { Airway malacia } \\
(\mathrm{N}=3) \\
\text { Aspiration }(\mathrm{N}=1) \\
\text { Urinary retention } \\
(\mathrm{N}=1) \\
\text { Connective tissue } \\
\text { disease }(\mathrm{N}=1) \\
22 \mathrm{q} \text { deletion } \\
(\mathrm{N}=1)\end{array}$ & $\begin{array}{l}\text { Diffuse PIG (45.8\%; } \\
\mathrm{N}=11) \\
\text { Patchy PIG (45.8\%, } \\
\mathrm{N}=11 \text { ) } \\
\text { Alveolar } \\
\text { simplification (79\%, } \\
\mathrm{N}=19) \\
\text { PAH (79\%, N=19) }\end{array}$ & $\begin{array}{l}\text { GGO (N=19) } \\
\text { Cysts (N=11) } \\
\text { Linear reticular } \\
\text { opacities } \\
(\mathrm{N}=3 / 22)\end{array}$ & $\begin{array}{l}\text { Off all respiratory } \\
\text { support }(\mathrm{N}=12) \\
\text { Supplemental } \\
\text { oxygen }(\mathrm{N}=8) \\
\text { Mechanical } \\
\text { ventilation }(\mathrm{N}=2) \\
\text { Lung } \\
\text { transplantation } \\
(\mathrm{N}=1) \\
\text { Died }(\mathrm{N}=1)\end{array}$ \\
\hline \multirow{11}{*}{$\begin{array}{l}\text { Weinman } \\
\text { et al, } 2018 \\
(10)\end{array}$} & \multirow{11}{*}{15} & 39 weeks & M & Birth & PFO, DTV & Yes & - & Diffuse PIG, PAH & GGO, cysts & Oxygen at rest \\
\hline & & 40 weeks & M & 3 months & PFO, PDA & Yes & $\begin{array}{l}\text { Aspiration, } \\
\text { autism, airway } \\
\text { malacia }\end{array}$ & $\begin{array}{l}\text { Patchy PIG, PAH, } \\
\text { Alveolar } \\
\text { simplification }\end{array}$ & $\begin{array}{l}\text { Architectural } \\
\text { distortion, } \\
\text { atelectasis }\end{array}$ & $\begin{array}{l}\text { Off respiratory } \\
\text { support }\end{array}$ \\
\hline & & 34 weeks & $\mathrm{F}$ & Birth & PFO, PVS & Yes & Seizures & $\begin{array}{l}\text { Diffuse PIG, PAH, PH } \\
\text { Alveolar } \\
\text { simplification }\end{array}$ & $\begin{array}{l}\text { GGO, cysts, } \\
\text { interlobular } \\
\text { septal } \\
\text { thickening }\end{array}$ & $\begin{array}{l}\text { Lung } \\
\text { transplantation } \\
\text { and oxygen at } \\
\text { rest }\end{array}$ \\
\hline & & 40 weeks & $\mathrm{F}$ & 3 months & Absent & No & - & Patchy PIG, PAH & GGO, cysts & $\begin{array}{l}\text { Oxygen with } \\
\text { sleep }\end{array}$ \\
\hline & & 32 weeks & $\mathrm{F}$ & Birth & Absent & Yes & Airway malacia & $\begin{array}{l}\text { Diffuse PIG, PAH, } \\
\text { Alveolar } \\
\text { simplification }\end{array}$ & $\begin{array}{l}\text { GGO, cysts, } \\
\text { architectural } \\
\text { distortion }\end{array}$ & $\begin{array}{l}\text { Off respiratory } \\
\text { support }\end{array}$ \\
\hline & & 29 weeks & $\mathrm{F}$ & Birth & PDA & No & Airway malacia & $\begin{array}{l}\text { Patchy PIG, PAH, PN } \\
\text { Alveolar } \\
\text { simplification }\end{array}$ & $\begin{array}{l}\text { Cysts, GGO, } \\
\text { interlobular } \\
\text { septal } \\
\text { thickening }\end{array}$ & $\begin{array}{l}\text { Mechanical } \\
\text { ventilation }\end{array}$ \\
\hline & & 39 weeks & M & Birth & Absent & Yes & Airway malacia & $\begin{array}{l}\text { Diffuse PIG, PAH, PH, } \\
\text { Alveolar } \\
\text { simplification }\end{array}$ & $\begin{array}{l}\text { Air trapping, } \\
\text { GGO }\end{array}$ & $\begin{array}{l}\text { Off respiratory } \\
\text { support }\end{array}$ \\
\hline & & 36 weeks & M & Birth & ASD & No & - & $\begin{array}{l}\text { Patchy PIG, PAH, } \\
\text { PN, PH, alveolar } \\
\text { simplification }\end{array}$ & $\begin{array}{l}\text { Cysts, GGO, } \\
\text { architectural } \\
\text { distortion }\end{array}$ & Oxygen at rest \\
\hline & & 29 weeks & M & Birth & PDA & No & - & $\begin{array}{l}\text { Patchy PIG, PAH, } \\
\text { CMV, alveolar } \\
\text { simplification }\end{array}$ & $\begin{array}{l}\text { Cysts, GGO, } \\
\text { architectural } \\
\text { distortion }\end{array}$ & $\begin{array}{l}\text { Off respiratory } \\
\text { support }\end{array}$ \\
\hline & & 36 weeks & M & Birth & TF & Yes & $\begin{array}{l}\text { Aspiration, septo- } \\
\text { optic dysplasia }\end{array}$ & $\begin{array}{l}\text { Patchy PIG, pleural } \\
\text { thickening, alveolar } \\
\text { simplification }\end{array}$ & $\begin{array}{l}\text { GGO, } \\
\text { atelectasis, PIE }\end{array}$ & $\begin{array}{l}\text { Off respiratory } \\
\text { support }\end{array}$ \\
\hline & & 37 weeks & M & Birth & Absent & Yes & $\mathrm{CDH}$ & $\begin{array}{l}\text { Diffuse PIG, PAH, PH, } \\
\text { Alveolar } \\
\text { simplification }\end{array}$ & $\begin{array}{l}\text { GGO, cysts, } \\
\text { interlobular } \\
\text { thickening }\end{array}$ & Died \\
\hline
\end{tabular}




\begin{tabular}{|c|c|c|c|c|c|c|c|c|c|c|}
\hline & & 30 weeks & M & Birth & ASD, PDA & Yes & - & $\begin{array}{l}\text { Diffuse PIG, PAH, } \\
\text { Alveolar } \\
\text { simplification }\end{array}$ & $\begin{array}{l}\text { Cysts, GGO, } \\
\text { interlobular } \\
\text { thickening }\end{array}$ & $\begin{array}{l}\text { Off respiratory } \\
\text { support }\end{array}$ \\
\hline & & 40 weeks & $\mathrm{F}$ & Birth & ASD, PDA & Yes & Single kidney & $\begin{array}{l}\text { Patchy PIG, PAH, } \\
\text { Alveolar } \\
\text { simplification }\end{array}$ & $\begin{array}{l}\text { GGO, } \\
\text { architectural } \\
\text { distortion, } \\
\text { atelectasis }\end{array}$ & Oxygen at rest \\
\hline & & 27 weeks & M & Birth & PDA & No & - & NA & $\begin{array}{l}\text { GGO, cysts, } \\
\text { interlobular } \\
\text { thickening }\end{array}$ & $\begin{array}{l}\text { Off respiratory } \\
\text { support }\end{array}$ \\
\hline & & 40 weeks & $\mathrm{F}$ & 1 month & $\begin{array}{l}\text { ASD, PDA, } \\
\text { ascending aortic } \\
\text { dilation }\end{array}$ & Yes & $\begin{array}{l}\text { CTD ACTA2 } \\
\text { mutation, } \\
\text { Airway malacia }\end{array}$ & NA & $\begin{array}{l}\text { Cysts, GGO, } \\
\text { architectural } \\
\text { distortion }\end{array}$ & $\begin{array}{l}\text { Off respiratory } \\
\text { support }\end{array}$ \\
\hline \multirow{9}{*}{$\begin{array}{l}\text { Seidl et al, } \\
2018 \text { (7) }\end{array}$} & \multirow{9}{*}{11} & Term & $\mathrm{F}$ & Birth & No & Yes & $\begin{array}{l}\text { Mucopolysaccha- } \\
\text { ridosis }\end{array}$ & NA & $\begin{array}{l}\text { Consolidation, } \\
\text { linear } \\
\text { opacities, } \\
\text { bronchiectasis }\end{array}$ & $\begin{array}{l}\text { Reduced, but } \\
\text { persistent } \\
\text { respiratory } \\
\text { symptoms, no } \\
\text { oxygen demand, } \\
\pm\end{array}$ \\
\hline & & Term & M & Birth & PDA & Yes & $\begin{array}{l}\text { Mucopolysaccha- } \\
\text { ridosis }\end{array}$ & $\begin{array}{l}\text { Diffuse PIG, } \\
\text { mild reduced } \\
\text { alveolarization }\end{array}$ & $\begin{array}{l}\text { GGO, } \\
\text { consolidation, } \\
\text { septal } \\
\text { thickening }\end{array}$ & $\begin{array}{l}\text { Reduced, but } \\
\text { persistent } \\
\text { respiratory } \\
\text { symptoms, no } \\
\text { oxygen demand, } \\
\pm\end{array}$ \\
\hline & & Term & M & Birth & VSD, PFO & Yes & $\begin{array}{l}\text { Brain (divided } \\
\text { plexus) }\end{array}$ & $\begin{array}{l}\text { Diffuse PIG, } \\
\text { moderate reduced } \\
\text { alveolarization }\end{array}$ & $\begin{array}{l}\text { Linear } \\
\text { opacities, } \\
\text { consolidation }\end{array}$ & Asymptomatic \\
\hline & & Term & M & Birth & Absent & Yes & - & $\begin{array}{l}\text { Diffuse PIG, } \\
\text { moderate reduced } \\
\text { alveolarization }\end{array}$ & $\begin{array}{l}\text { GGO, mosaic } \\
\text { attenuation, } \\
\text { linear opacity, } \\
\text { consolidation, } \\
\text { architectural } \\
\text { distortion }\end{array}$ & Died \\
\hline & & 34 weeks & M & Birth & Absent & No & $\begin{array}{l}\text { Encephalopathy, } \\
\text { hepatic cysts }\end{array}$ & $\begin{array}{l}\text { Diffuse PIG, } \\
\text { mild reduced } \\
\text { alveolarization }\end{array}$ & $\begin{array}{l}\text { Hyperinflated } \\
\text { 2ndary } \\
\text { lobule, septal } \\
\text { thickening }\end{array}$ & $\begin{array}{l}\text { Reduced, but } \\
\text { persistent } \\
\text { respiratory } \\
\text { symptoms, no } \\
\text { oxygen demand, } \\
\pm \pm\end{array}$ \\
\hline & & Term & $\mathrm{F}$ & Birth & Absent & Yes & - & $\begin{array}{l}\text { Diffuse PIG, } \\
\text { mild reduced } \\
\text { alveolarization }\end{array}$ & $\begin{array}{l}\text { GGO, } \\
\text { consolidation, } \\
\text { mosaic } \\
\text { attenuation, } \\
\text { linear } \\
\text { opacities }\end{array}$ & Asymptomatic \\
\hline & & 30 weeks & M & Birth & PDA & No & Hypoglycemia & NA & $\begin{array}{l}\text { GGO, septal } \\
\text { thickening, } \\
\text { crazy paving } \\
\text { pattern, } \\
\text { linear opacity, } \\
\text { hyperinflated } \\
\text { secondary } \\
\text { lobule }\end{array}$ & $\begin{array}{l}\text { Reduced, but } \\
\text { persistent } \\
\text { respiratory } \\
\text { symptoms, no } \\
\text { oxygen demand }\end{array}$ \\
\hline & & Term & $\mathrm{F}$ & Birth & Absent & Yes & - & $\begin{array}{l}\text { Diffuse PIG, } \\
\text { severe reduced } \\
\text { alveolarization }\end{array}$ & $\begin{array}{l}\text { GGO, linear } \\
\text { opacity, } \\
\text { bronchial wall } \\
\text { thickening }\end{array}$ & Asymptomatic \\
\hline & & Term & M & 7 weeks & AVSD & Yes & . & $\begin{array}{l}\text { Patchy PIG, } \\
\text { moderate reduced } \\
\text { alveolarization }\end{array}$ & $\begin{array}{l}\text { GGO, mosaic } \\
\text { attenuation, } \\
\text { septal } \\
\text { thickening, } \\
\text { emphysema }\end{array}$ & Asymptomatic \\
\hline & & Term & $\mathrm{F}$ & Birth & $\begin{array}{l}\text { VSD, ASD } \\
\text { hypoplastic } \\
\text { pulmonary } \\
\text { arterial system }\end{array}$ & Yes & $\begin{array}{l}\text { Renal failure, } \\
\text { megaureter (left) }\end{array}$ & $\begin{array}{l}\text { Diffuse PIG, } \\
\text { severe reduced } \\
\text { alveolarization }\end{array}$ & NA & Asymptomatic \\
\hline
\end{tabular}




\begin{tabular}{|c|c|c|c|c|c|c|c|c|c|c|}
\hline & & Term & M & Birth & $\begin{array}{l}\text { ASD, VSD, } \\
\text { coarctation }\end{array}$ & Yes & $\begin{array}{l}\text { Heterotaxy } \\
\text { syndrome (heart, } \\
\text { lung, abdomen) }\end{array}$ & $\begin{array}{l}\text { Diffuse PIG, } \\
\text { severe reduced } \\
\text { alveolarization }\end{array}$ & $\begin{array}{l}\text { GGO, } \\
\text { consolidation }\end{array}$ & $\begin{array}{l}\text { Reduced, but } \\
\text { persistent } \\
\text { respiratory } \\
\text { symptoms, no } \\
\text { oxygen demand, } \\
\pm \pm\end{array}$ \\
\hline $\begin{array}{l}\text { Still et al, } \\
2018 \text { (17) }\end{array}$ & 1 & Term & $\mathrm{F}$ & Birth & Absent & Yes & - & $\begin{array}{l}\text { Patchy PIG, } \\
\text { Alveolar } \\
\text { simplification } \\
\text { (moderate), PAH }\end{array}$ & $\begin{array}{l}\text { Diffuse mosaic } \\
\text { attenuation, } \\
\text { right } \\
\text { pulmonary } \\
\text { artery } \\
\text { enlargement }\end{array}$ & $\begin{array}{l}\text { On low flow } \\
\text { oxygen, } \\
\text { multidrug } \\
\text { regimen for } \\
\text { persistent } \\
\text { pulmonary } \\
\text { hypertension }\end{array}$ \\
\hline $\begin{array}{l}\text { Demirel } \\
\text { et al, } 2018 \\
\text { (18) }\end{array}$ & 1 & Term & $\mathrm{F}$ & $\begin{array}{l}\text { Shortly after } \\
\text { birth }\end{array}$ & $\begin{array}{l}\text { PDA (large), } \\
\text { secundum ASD* }\end{array}$ & Yes & $\begin{array}{l}\text { Filamin A protein } \\
\text { deficiency, } \\
\text { Bronchomalacia } \\
\text { (LLL) }\end{array}$ & $\begin{array}{l}\text { Patchy PIG (mild), } \\
\text { moderate alveolar } \\
\text { simplification and } \\
\text { hyperinflation }\end{array}$ & $\begin{array}{l}\text { Findings most } \\
\text { consistent } \\
\text { with Filamin A } \\
\text { deficiency } * *\end{array}$ & $\begin{array}{l}\text { Supplemental } \\
\text { oxygen, } \\
\text { multidrug } \\
\text { regimen for } \\
\text { pulmonary } \\
\text { hypertension, } \\
\text { steroids }\end{array}$ \\
\hline \multirow{5}{*}{$\begin{array}{l}\text { Deutsch } \\
\text { et al, } 2016 \\
\text { (19) }\end{array}$} & \multirow{5}{*}{5} & 38 & M & Birth & Absent & NA & - & $\begin{array}{l}\text { PIG (pattern } \\
\text { distribution NA), } \\
\text { lung growth } \\
\text { abnormality }\end{array}$ & NA & $\begin{array}{l}\text { Persistent } \\
\text { tachypnea, } \\
\text { cough }\end{array}$ \\
\hline & & 37 & M & Birth & Absent & NA & $\begin{array}{l}\text { Severe } \\
\text { chylothorax }\end{array}$ & $\begin{array}{l}\text { PIG (pattern } \\
\text { distribution NA), } \\
\text { lung growth } \\
\text { abnormality }\end{array}$ & NA & Asymptomatic \\
\hline & & 41 & $\mathrm{~F}$ & Birth & $\begin{array}{l}\text { Total anomalous } \\
\text { pulmonary } \\
\text { venous return/ } \\
\text { vein stenosis }\end{array}$ & Yes & - & $\begin{array}{l}\text { PIG (pattern NA), } \\
\text { lymphangiectasia, } \\
\text { PAH }\end{array}$ & NA & Died \\
\hline & & 35 & $\mathrm{~F}$ & Birth & $\begin{array}{l}\text { Double outlet } \\
\text { right ventricle }\end{array}$ & Yes & VACTERL & $\begin{array}{l}\text { PIG (pattern } \\
\text { NA), lung growth } \\
\text { abnormality, PAH }\end{array}$ & NA & $\begin{array}{l}\text { Intermittent } \\
\text { asthma }\end{array}$ \\
\hline & & 36 & M & Birth & Absent & Yes & - & $\begin{array}{l}\text { PIG (pattern } \\
\text { NA), lung growth } \\
\text { abnormality, PAH }\end{array}$ & NA & Asymptomatic \\
\hline $\begin{array}{l}\text { Sanchez-de- } \\
\text { Toledo et al, } \\
2015 \text { (20) }\end{array}$ & 1 & Term & NA & Birth & $\begin{array}{l}\text { D-transposition } \\
\text { of great arteries } \\
\text { with intact } \\
\text { ventricular } \\
\text { septum }\end{array}$ & Yes & - & PIG & NA & $\begin{array}{l}\text { Successful } \\
\text { corrective } \\
\text { surgery, } \\
\text { Asymptomatic }\end{array}$ \\
\hline $\begin{array}{l}\text { Simons et } \\
\text { al, } 2014 \\
(21)\end{array}$ & 1 & NA & $\mathrm{F}$ & 1 month & $\begin{array}{l}\text { ASD, massive } \\
\text { window duct } \\
\text { with right } \\
\text { ventricular } \\
\text { hypertrophy and } \\
\text { pulmonary trunk } \\
\text { dilation }\end{array}$ & NA & Aniridia & $\begin{array}{l}\text { PIG, alveolar } \\
\text { simplification }\end{array}$ & NA & $\begin{array}{l}\text { Successful } \\
\text { corrective } \\
\text { surgery, } \\
\text { Asymptomatic }\end{array}$ \\
\hline $\begin{array}{l}\text { Ehsan et al, } \\
2014 \text { (22) }\end{array}$ & 1 & Term & M & Birth & Absent & NA & - & $\begin{array}{l}\text { Diffuse PIG } \\
\text { Alveolar } \\
\text { simplification } \\
\text { (diffuse) }\end{array}$ & GGO (diffuse) & $\begin{array}{l}\text { Asymptomatic, } \\
\text { no oxygen } \\
\text { demand, } \\
\text { although FVC } \\
\text { remained } \\
\text { significantly } \\
\text { reduced } \\
\text { compared to } \\
\text { healthy control } \\
\text { patients }\end{array}$ \\
\hline $\begin{array}{l}\text { Ross et al, } \\
2014 \text { (23) }\end{array}$ & 1 & 34 & M & Birth & $\begin{array}{l}\text { Pulmonary valve } \\
\text { abnormalities }\end{array}$ & No & $\begin{array}{l}\text { Noonan syndrome } \\
\text { (positive test for } \\
\text { heterozygous } \\
\text { G60A mutation in } \\
\text { PTPN11) }\end{array}$ & $\begin{array}{l}\text { Diffuse PIG, } \\
\text { Alveolar growth } \\
\text { abnormality/ } \\
\text { simplification, } \\
\text { Mild acute } \\
\text { inflammation }\end{array}$ & $\begin{array}{l}\text { Septal } \\
\text { thickening, } \\
\text { Extensive } \\
\text { dependent } \\
\text { airspace } \\
\text { opacities, } \\
\text { Small pleural } \\
\text { effusions }\end{array}$ & $\begin{array}{l}\text { On nocturnal } \\
\text { supplemental } \\
\text { oxygen }\end{array}$ \\
\hline
\end{tabular}




\begin{tabular}{|c|c|c|c|c|c|c|c|c|c|c|}
\hline $\begin{array}{l}\text { Alkhorayyef } \\
\text { et al, } 2013 \\
\text { (16) }\end{array}$ & 1 & Term & M & Birth & $\begin{array}{l}\text { Severe } \\
\text { hypertrophic } \\
\text { cardiomyopathy, } \\
\text { PFO }\end{array}$ & Yes & - & Diffuse PIG & $\begin{array}{l}\text { CT none done } \\
\text { Chest X-ray: } \\
\text { bilateral GGO }\end{array}$ & Died \\
\hline \multirow{2}{*}{$\begin{array}{l}\text { Radman } \\
\text { et al, } 2012 \\
\text { (14) }\end{array}$} & \multirow[t]{2}{*}{2} & Term & $\mathrm{F}$ & Birth & $\begin{array}{l}\text { D-transposition } \\
\text { of great arteries } \\
\text { with intact } \\
\text { ventricular } \\
\text { septum, PFO, } \\
\text { and large PDA }\end{array}$ & Yes & - & Patchy PIG, PAH & NA & Asymptomatic \\
\hline & & Term & M & Birth & $\begin{array}{l}\text { Heterotaxy } \\
\text { with complex } \\
\text { cardiovascular } \\
\text { abnormalities }\end{array}$ & Yes & - & $\begin{array}{l}\text { Diffuse PIG, PAH } \\
\text { (minimal) }\end{array}$ & NA & $\begin{array}{l}\text { On supplemental } \\
\text { oxygen }\end{array}$ \\
\hline $\begin{array}{l}\text { King et al, } \\
2011 \text { 915) }\end{array}$ & 1 & Term & M & Birth & Large PDA, PFO & Yes & - & $\begin{array}{l}\text { Patchy PIG, severe } \\
\text { lung growth } \\
\text { abnormality, PAH } \\
\text { (mild) }\end{array}$ & NA & Died \\
\hline $\begin{array}{l}\text { Smets et al, } \\
2011 \text { (23) \& } \\
2004 \text { (24) }\end{array}$ & 1 & Term & & Birth & Absent & Yes & $\begin{array}{l}\text { Hunter syndrome } \\
\text { (mucopolysaccha- } \\
\text { ridosis type II) }\end{array}$ & Diffuse PIG & $\begin{array}{l}\text { Architectural } \\
\text { distortion, } \\
\text { linear } \\
\text { opacities, } \\
\text { areas of } \\
\text { hyperinflation } \\
\text { and GGO }\end{array}$ & $\begin{array}{l}\text { Frequent upper } \\
\text { respiratory } \\
\text { infections, } \\
\text { Lung function } \\
\text { tests: severe } \\
\text { combined } \\
\text { obstructive/ } \\
\text { restrictive } \\
\text { pattern }\end{array}$ \\
\hline $\begin{array}{l}\text { Castillo et } \\
\text { al, 2010 } \\
(12)\end{array}$ & 1 & 37 & M & Birth & Absent & NA & - & $\begin{array}{l}\text { Patchy PIG, } \\
\text { Severe alveolar } \\
\text { growth abnormality, } \\
\text { Mild pleural } \\
\text { thickening }\end{array}$ & $\begin{array}{l}\text { GGO (diffuse), } \\
\text { prominent } \\
\text { interstitial } \\
\text { opacities, } \\
\text { multiple } \\
\text { scattered } \\
\text { cystic spaces }\end{array}$ & Asymptomatic \\
\hline $\begin{array}{l}\text { Lanfranchi } \\
\text { et al, } 2010 \\
\text { (26) }\end{array}$ & 1 & 31 & M & $\begin{array}{l}18 \text { days of } \\
\text { life }\end{array}$ & Absent & NA & - & Diffuse PIG & $\begin{array}{l}\text { Diffuse coarse } \\
\text { reticular } \\
\text { opacities }\end{array}$ & Asymptomatic \\
\hline \multirow{2}{*}{$\begin{array}{l}\text { Orlando } \\
\text { et al, } 2005 \\
\text { (27) }\end{array}$} & \multirow{2}{*}{$\begin{array}{l}2 \\
\text { (identical } \\
\text { twins) }\end{array}$} & 31 & M & Birth & Absent & No & - & Diffuse PIG & $\begin{array}{l}\text { GGO, septal } \\
\text { thickening }\end{array}$ & Asymptomatic \\
\hline & & 31 & M & Birth & Absent & No & - & Diffuse PIG & $\begin{array}{l}\text { GGO, septal } \\
\text { thickening }\end{array}$ & Asymptomatic \\
\hline \multirow{7}{*}{$\begin{array}{l}\text { Canakis et } \\
\text { al, } 2002 \text { (4) }\end{array}$} & \multirow{7}{*}{7} & 33 & M & 5 days & Absent & NA & - & Diffuse PIG & $\begin{array}{l}\text { Not done } \\
\text { at initial } \\
\text { presentation }\end{array}$ & $\begin{array}{l}\text { Mild intermittent } \\
\text { bronchospasm }\end{array}$ \\
\hline & & 38 & M & 1 day & Absent & NA & - & Diffuse PIG & $\begin{array}{l}\text { GGO, patchy } \\
\text { (performed at } \\
7 \text { months age) }\end{array}$ & $\begin{array}{l}\text { Mild intermittent } \\
\text { bronchospasm }\end{array}$ \\
\hline & & 40 & M & 4 weeks & Absent & NA & - & Diffuse PIG & $\begin{array}{l}\text { GGO, patchy } \\
\text { (performed at } \\
2 \text { years of age) }\end{array}$ & Asymptomatic \\
\hline & & 33 & M & 1 day & Absent & NA & _- & Diffuse PIG & NA & $\begin{array}{l}\text { On supplemental } \\
\text { oxygen }\end{array}$ \\
\hline & & 29 & $\mathrm{~F}$ & 1 day & Absent & NA & - & Diffuse PIG & NA & Asymptomatic \\
\hline & & 40 & M & 1 day & Absent & NA & - & Diffuse PIG & NA & Asymptomatic \\
\hline & & 25 & M & 1 day & Absent & NA & - & Diffuse PIG & NA & $\begin{array}{l}\text { Died (cor } \\
\text { pulmonale) }\end{array}$ \\
\hline
\end{tabular}

Abbreviations: $\mathrm{PIG}=$ pulmonary interstitial glycogenosis, $\mathrm{N}=$ number of patients, $\mathrm{GA}=$ gestational age, $\mathrm{CHD}=$ congenital heart disease, $\mathrm{PHN}=$ pulmonary arterial hypertension, HRCT= high-resolution computed tomography, HLHS=hypoplastic left heart syndrome, PVS= pulmonary vein stenosis, ASD=atrial septal defect, VSD=ventricular septal defects, $\mathrm{PDA}=$ patent ductus arteriosus, $\mathrm{TF}=$ Tetralogy of Fallot, $\mathrm{NA}=$ not available, $\mathrm{PAH}=$ pulmonary artery hypertrophy, $\mathrm{GGO}=\mathrm{ground}$ glass opacities, $\mathrm{PFO}=$ patent foramen ovale, $\mathrm{DTV}=$ dysplastic tricuspid valve, $\mathrm{PH}=$ pulmonary hemorrhage, $\mathrm{PN}=$ pneumonia, $\mathrm{CMV}=$ cytomegalovirus, $\mathrm{CDH}=$ congenital diaphragmatic hernia, $C T D=c o n n e c t i v e$ tissue disease, $A V S D=$ atrial-ventricular septal defects, LLL=left lower lobe, VACTERL= vertebral defects, anal atresia, cardiac defects, tracheo-esophageal fistula, renal anomalies, and limb abnormalities, $\mathrm{FVC}=$ forced vital capacity.

* large patent PDA, secundum atrial septal defect with left to right shunting, associated left atrial, right atrial and right ventricular enlargement, and pulmonary insufficiency with right ventricular hypertrophy. Moderate to severe pulmonary arterial hypertension.

**diffuse pulmonary hyperinflation, pruning of the peripheral pulmonary vasculature, and patchy areas of atelectasis.

\pm recurrent respiratory infections, reduced exercise tolerance

\pm \pm tachypnea at rest 
PIG was first described in 2002 by Canakis et al (4). The definitive diagnosis is made by histologic examination. Histologic features on lung biopsy are characterized by expansion of pulmonary interstitium by round to ovoidshaped mesenchymal cells with pale cytoplasm (fig. 1A $\& 1 B)$ due to glycogen accumulation demonstrable by periodic acid-Schiff positivity (diastase sensitive) on light microscopy. However, the presence of glycogen is best identified on electron microscopy (EM). The ultrastructural examination showed poorly differentiated interstitial mesenchymal cells with vesicular nuclei and cytoplasm containing sparse organelles and abundant monoparticulate glycogen (fig. 1C \& 1D). Some PIG cells showed features of differentiation toward the fibroblast lineage differentiation (2).

Additionally, 28 cases of PIG in association with a spectrum of lung or cardiac disorders have been reported recently by Cutz et al. In this paper, we described four clinicopathologic subgroups including comorbidities, imaging and outcome (summarized in Table 2). As previously reported $(3,6,8)$, we found that PIG is most commonly

Table 2: List of Cases of PIG Reported by Cutz et al; Patients' Gender, Gestational Age, Comorbidities, Histopathology, Imaging Findings, And Clinical Outcome at Last Follow-Up

\begin{tabular}{|c|c|c|c|c|c|c|c|}
\hline Category & Patient & $\begin{array}{l}\text { GA, preterm, } \\
\text { or term }\end{array}$ & Sex & Comorbidity & Lung histopathology & Chest HRCT findings & Outcome \\
\hline \multirow{8}{*}{1} & 1 & Term & M & Pulmonary hypertension & AGA, diffuse PIG, PAH & NA & Died \\
\hline & 2 & Term & $\mathrm{F}$ & - & AGA, patchy PIG & NA & NA \\
\hline & 3 & 32 weeks & M & - & AGA (severe), Diffuse PIG & NA & NA \\
\hline & 4 & Term & M & Pulmonary hypertension & AGA, Diffuse PIG, PAH & GGO (diffuse) & Died \\
\hline & 5 & Term & M & Pulmonary hypertension & AGA, Patchy PG, PAH & GGO & Died \\
\hline & 6 & 33 weeks & $\mathrm{F}$ & $\begin{array}{l}\text { Pulmonary } \\
\text { lymphangiectasis }\end{array}$ & $\begin{array}{l}\text { AGA, Diffuse PIG, } \\
\text { lymphangiectasia }\end{array}$ & $\begin{array}{l}\text { Diffuse septal } \\
\text { thickening, bilateral } \\
\text { pleural effusion }\end{array}$ & NA \\
\hline & 7 & Term & M & Congenital chylothorax & NA & NA & Died \\
\hline & 8 & Term & $\mathrm{F}$ & $\begin{array}{l}\text { Pulmonary hypertension, } \\
\text { arthrogryposis }\end{array}$ & AGA, patchy PIG, PAH & NA & Died \\
\hline \multirow{6}{*}{ II } & 9 & Term & M & $\begin{array}{l}\text { PDA, dilated } \\
\text { cardiomyopathy, } \\
\text { pulmonary hypertension }\end{array}$ & Patchy PIG, PAH (mild) & GGO (focal) & Died \\
\hline & 10 & Term & $\mathrm{F}$ & $\begin{array}{l}\text { Complex cardiac } \\
\text { abnormalities (right atrial } \\
\text { isomerism, dextrocardia, } \\
\text { AVSD, DORV, hypoplastic } \\
\text { RPA), pulmonary } \\
\text { hypertension }\end{array}$ & $\begin{array}{l}\text { Focal PIG, } \\
\text { lymphangiectasis, PAH } \\
\text { (moderate) }\end{array}$ & GGO (patchy) & Died \\
\hline & 11 & Term & M & $\begin{array}{l}\text { Hypoplastic left heart } \\
\text { syndrome }\end{array}$ & Focal PIG, AGA, PAH (mild) & GGO & Died \\
\hline & 12 & 36 weeks & $\mathrm{F}$ & $\begin{array}{l}\text { Noonan syndrome } \\
\text { with hypertrophic } \\
\text { cardiomyopathy }\end{array}$ & $\begin{array}{l}\text { Patchy PIG, } \\
\text { lymphangiectasis, PAH }\end{array}$ & $\begin{array}{l}\text { Diffuse septal } \\
\text { thickening, bilateral } \\
\text { pleural effusion }\end{array}$ & Died \\
\hline & 13 & Term & $\mathrm{F}$ & $\begin{array}{l}\text { ASD, VSD, pulmonary } \\
\text { hypertension, aspiration, } \\
\text { pneumonia }\end{array}$ & Diffuse PIG, PAH (mild) & $\begin{array}{l}\text { Diffuse lung } \\
\text { interstitial disease }\end{array}$ & Died \\
\hline & 14 & & M & TAPVD & Diffuse PIG, AGA & $\begin{array}{l}\text { GGO (diffuse, mild), } \\
\text { septal thickening } \\
\text { (patchy) }\end{array}$ & $\begin{array}{l}\text { Successful corrective } \\
\text { surgery }\end{array}$ \\
\hline \multirow{5}{*}{ III } & 15 & Term & M & Pulmonary hypertension & $\begin{array}{l}\text { Hyperplasia of PNE cells, } \\
\text { diffuse PIG, }\end{array}$ & $\begin{array}{l}\text { "crazy paving" } \\
\text { appearance }\end{array}$ & $\begin{array}{l}\text { Off all respiratory } \\
\text { support }\end{array}$ \\
\hline & 16 & Term & M & $\begin{array}{l}\text { Pulmonary } \\
\text { lymphangiectasia }\end{array}$ & $\begin{array}{l}\text { Hyperplasia of PNE } \\
\text { cells, PIG, AGA, } \\
\text { lymphangiectasia, }\end{array}$ & $\begin{array}{l}\text { GGO, septal } \\
\text { thickening, patchy } \\
\text { hyperinflation }\end{array}$ & $\begin{array}{l}\text { Off all respiratory } \\
\text { support }\end{array}$ \\
\hline & 17 & 30 weeks & $M$ & $\begin{array}{l}\text { ASD, VSD, airway malacia, } \\
\text { autism }\end{array}$ & $\begin{array}{l}\text { Hyperplasia of PNE cells, } \\
\text { PIG, PAH (mild) }\end{array}$ & $\begin{array}{l}\text { GGO, basal } \\
\text { hyperinflation }\end{array}$ & $\begin{array}{l}\text { Off all respiratory } \\
\text { support }\end{array}$ \\
\hline & 18 & 36 weeks & $\mathrm{F}$ & Pulmonary hypertension & $\begin{array}{l}\text { Hyperplasia of PNE cells, } \\
\text { patchy PIG, PAH (mild) }\end{array}$ & NA & NA \\
\hline & 19 & Term & $\mathrm{F}$ & Pulmonary hypertension & $\begin{array}{l}\text { Hyperplasia of PNE cells, } \\
\text { patchy PIG }\end{array}$ & NA & NA \\
\hline
\end{tabular}




\begin{tabular}{|c|c|c|c|c|c|c|c|}
\hline \multirow[t]{9}{*}{ IV } & 20 & Term & $\mathrm{F}$ & - & $\begin{array}{l}\text { CPAM type I (large cyst } \\
\text { type), patchy PIG }\end{array}$ & $\begin{array}{l}\text { Multicystic lesion } \\
\text { with air-filled, thin- } \\
\text { walled spaces of } \\
\text { large size in in LLL }\end{array}$ & $\begin{array}{l}\text { Asymptomatic after } \\
\text { surgical treatment }\end{array}$ \\
\hline & 21 & Term & $\mathrm{F}$ & Chiari malformation & $\begin{array}{l}\text { CPAM type I (large cyst } \\
\text { type), diffuse PIG }\end{array}$ & $\begin{array}{l}\text { Multicystic lesion } \\
\text { with air-filled, thin- } \\
\text { walled spaces of } \\
\text { large size in RML } \\
\text { \& LLL }\end{array}$ & $\begin{array}{l}\text { Asymptomatic after } \\
\text { surgical treatment }\end{array}$ \\
\hline & 22 & Term & $\mathrm{F}$ & Renal dysplasia & $\begin{array}{l}\text { CPAM type I with systemic } \\
\text { arterial supply (hybrid } \\
\text { lesion), focal PIG }\end{array}$ & $\begin{array}{l}\text { Large air-filled cystic } \\
\text { lesion with systemic } \\
\text { vascular supply }\end{array}$ & $\begin{array}{l}\text { Asymptomatic } \\
\text { after surgical } \\
\text { treatment }\end{array}$ \\
\hline & 23 & 31 weeks & M & - & $\begin{array}{l}\text { CPAM type I with systemic } \\
\text { arterial supply (hybrid } \\
\text { lesion), focal PIG }\end{array}$ & $\begin{array}{l}\text { Fluid and air-filled } \\
\text { large cystic lesion } \\
\text { with systemic } \\
\text { vascular supply }\end{array}$ & $\begin{array}{l}\text { Asymptomatic after } \\
\text { surgical treatment }\end{array}$ \\
\hline & 24 & Term & M & - & $\begin{array}{l}\text { CPAM type I (large cyst- } \\
\text { type), focal PIG }\end{array}$ & $\begin{array}{l}\text { Large air-filled cystic } \\
\text { lesion }\end{array}$ & $\begin{array}{l}\text { Asymptomatic after } \\
\text { surgical treatment }\end{array}$ \\
\hline & 25 & Term & $\mathrm{F}$ & CHD & CLE with focal PIG & RML hyperinflation & Cardiac complication \\
\hline & 26 & Term & M & - & CLE with focal PIG & RML hyperinflation & Asymptomatic \\
\hline & 27 & Term & M & - & CLE with diffuse PIG & RUL overinflation & $\begin{array}{l}\text { Asymptomatic after } \\
\text { surgical treatment }\end{array}$ \\
\hline & 28 & Term & M & - & CLE with diffuse PIG & LUL hyperinflation & $\begin{array}{l}\text { Asymptomatic after } \\
\text { surgical treatment }\end{array}$ \\
\hline
\end{tabular}

Abbreviations: $\mathrm{PIG}=$ pulmonary interstitial glycogenosis, $\mathrm{GA}=$ gestational age, $\mathrm{AGA}=$ alveolar growth abnormality, $\mathrm{HRCT}=$ high-resolution computed tomography, $A S D=$ atrial septal defect, $V S D=$ ventricular septal defects, $P D A=$ patent ductus arteriosus, $N A=n o t$ available, $P A H=$ pulmonary artery hypertrophy, DORV=double outlet right ventricle, hypoplastic RPA=hypoplastic right pulmonary artery, GGO=ground glass opacities, $A V S D=$ atrial-ventricular septal defects, $T A P V D=$ Total Anomalous Pulmonary Venous Drainage, CPAM=congenital pulmonary

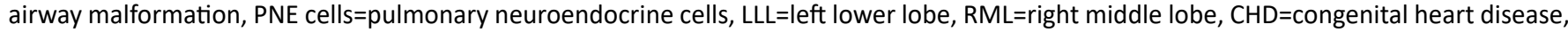
$\mathrm{CLE}=$ congenital lobar emphysema/hyperinflation, $\mathrm{RUL}=$ right upper lobe, LUL=left upper lobe.

associated with lung/alveolar growth abnormality, with or without pulmonary arterial hypertension. The mean age at time of biopsy was 10.3 weeks (range 16 days- 6 months). This group of patients presented with respiratory distress in the first weeks of life. Most patients in our series had pulmonary hypertension. Chest imaging studies revealed variable changes such as diffuse lung interstitial thickening and ground-glass opacities. The lung biopsies showed patchy or diffuse PIG changes, and abnormal alveolarization manifested by alveolar enlargement and simplification. This group of patients had a high mortality rate with 6 of the 9 infants dying of respiratory failure within few weeks of presentation despite conventional treatment. Most infants in this group developed refractory pulmonary hypertension.

The second subgroup represented patients with PIG associated with congenital heart diseases (CHD) (including hypoplastic left-heart syndrome, hypertrophic cardiomyopathy in patient with Noonan syndrome). Chest imaging studies showed variable non-specific changes (including ground glass opacity, septal thickening). The mean age at lung biopsy was 2.4 weeks (5 days-6weeks). The PIG changes on light microscopy were focal to patchy. Most infants died of complications despite corrective surgery.
The third category consisted of a specific group of patients with combined PIG and hyperplasia of pulmonary neuroendocrine cells (PNEC), referred to as neuroendocrine hyperplasia of infancy-like (NEHI-like). Infants of this group were either pre-term or full-term, mostly presented with tachypnea and wheezing between 3 and 10 weeks of age. Chest imaging studies revealed variable changes including "crazy paving" appearance, bilateral ground-glass opacities or basal hyperinflation. Lung biopsies demonstrated patchy to diffuse PIG, and prominent hyperplasia of PNEC (identified with immunohistochemistry studies). The patients of this group were mostly asymptomatic over time with normal lung function or persistent mild obstructive defects. The clear significance and etiology of this combined pathology is unknown. PNEC system has multifaceted roles including lung development, neonatal adaptation as airway oxygen sensors, and postnatal airway homeostasis as guardians of a stem cell niche (9). Hyperplasia of PNEC has been identified in several perinatal pediatric lung disorders including bronchopulmonary dysplasia, neuroendocrine hyperplasia of infancy, central hypoventilation syndrome, Sudden Infant Death Syndrome, and cystic fibrosis (9).

The fourth and final group consisted of cases of congenital lung malformation with coincident PIG. This included 5 patients with CPAM type 1 (large cyst type) and 4 patients with congenital lobar emphysema/hyperinflation 
(CLE). All patients presented with respiratory distress, soon after birth for CPAM, and between 4 weeks and 8 months of age for CLE cases. Chest imaging studies demonstrated changes related to the underlying diseases; localized cystic lesion in patients with CPAM, and lobar emphysema/hyperinflation in patients with CLE. The mean age at time of biopsy was 8.8 weeks for CPAM (range 3 days - 4 weeks), and 11.6 weeks for CLE (range 4 weeks- 8 months). Pathologic examination confirmed the diagnosis of CPAM type 1 or CLE. In addition, it showed patchy PIG changes in both the lesion and adjacent $<<$ normal $>>$ areas of pulmonary tissue. All patients underwent lobectomy of the affected lung. Except for one case, who had CLE and congenital heart disease, patient with CPAM and CLE all recovered post-surgery and were asymptomatic on followup.

In summary, the spectrum of disorders in association with PIG reported by us (2) is diverse and is quite like that reported by Langston et al (3), Liptzin et al. (6), Dishop M. (8), and Weinman (10). We found that PIG is most commonly seen in association with lung alveolar growth abnormality (alveolar simplification) and / or cardiovascular diseases. We have also described additional cases of PIG associated with persistent pulmonary hypertension with or without CHD, cardiovascular disease, Noonan syndrome (23), and congenital lobular emphysema (28). In addition, we have reported a new association of PIG with other lung disorders including NEHI-like, and CPAM type 1 (large cyst type). Similarly, to previous published studies by Liptzin et al. (6), Weinman et al (10), Deutsch et al. (11), Castillo et al. (12), and Lee E. (13), we noticed that the imaging of PIG is variable, non-specific (including diffuse ground-glass opacities, hyperinflation, and cystic spaces), and is likely affected by the presence of coexisting lung disorders. Based on available literature, PIG is considered to have a favorable prognosis, although clinical outcome is dependent on the severity of any associated lung disorders or other comorbidities. In our series, we found that the mortality rate was high when PIG coincided with lifethreatening comorbidities (including severe lung growth abnormality, complex cardiovascular disease). This note was also emphasized by multiple previously published reports $(6,11,12,14,15,16)$. In the review by Deutsch et al (16), no mortality occurred among the six cases of diffuse/ isolated PIG, although respiratory symptoms persist in most patients. Given no radiographic patterns, genetic findings, or biomarkers were characteristic of PIG, the lung biopsy remains the gold standard for diagnosis.

Finally, the precise nature and clinical significance of PIG is unknown. While the pathology demonstrated poorly differentiated interstitial mesenchymal cells, there is a debate whether PIG is a primary developmental lung disorder or a reactive process to abnormal lung development and injury. Our finding of a close association of PIG with different lung developmental disorders and comorbid cardiac developmental diseases favors a defect in interstitial fibroblast differentiation. Further studies are required to define the precise pathogenesis and significance of PIG and its impact on concurrent disease processes.

\section{References}

1. Deutsch GH, Young LR, Deterding RR, et al. ChILD Research Cooperative. Diffuse lung disease in young children: application of a novel classification scheme. Am J Respir Crit Care Med. 2007 Dec 1; 176(11): 1120-8.

2. Cutz E, Chami R, Dell S, et al. Pulmonary interstitial glycogenosis associated with a spectrum of neonatal pulmonary disorders. Hum Pathol. 2017 Oct; 68: 154-165.

3. Langston C, Dishop MK. Diffuse lung disease in infancy: a proposed classification applied to 259 diagnostic biopsies. Pediatr Dev Pathol. 2009 Nov-Dec; 12(6): 421-37.

4. Canakis AM, Cutz E, Manson D, et al. Pulmonary interstitial glycogenosis: a new variant of neonatal interstitial lung disease. Am J Respir Crit Care Med. 2002 Jun 1; 165(11): 1557-65.

5. Deterding RR. Infants and Young Children with Children's Interstitial Lung Disease. Pediatr Allergy Immunol Pulmonol. 2010 Mar; 23(1): 25-31.

6. Liptzin DR, Baker CD, Darst JR, et al. Pulmonary interstitial glycogenosis: Diagnostic evaluation and clinical course. Pediatr Pulmonol. 2018 Dec; 53(12): 1651-1658.

7. Seidl E, Carlens J, Reu S, et al. Pulmonary interstitial glycogenosis - A systematic analysis of new cases. Respir Med. 2018 Jul; 140: 11-20.

8. Dishop MK. Diagnostic Pathology of Diffuse Lung Disease in Children. Pediatr Allergy Immunol Pulmonol. 2010 Mar; 23(1): 69-85.

9. Cutz E, Yeger H, Pan J. Pulmonary Neuroendocine Cell System in Pediatric Lung Disease - Recent Advances. Pediatr Dev Pathol. 2007 Nov-Dec; 10(6): 419-35.

10. Weinman JP, White CJ, Liptzin DR, et al. High-resolution CT findings of pulmonary interstitial glycogenosis. Pediatr Radiol. 2018 Aug; 48(8): 1066-1072.

11. Deutsch GH, Young LR. Pulmonary interstitial glycogenosis: words of caution. Pediatr Radiol. 2010 Sep; 40(9): 1471-5.

12. Castillo M, Vade A, Lim-Dunham JE, et al. Pulmonary interstitial glycogenosis in the setting of lung growth abnormality: radiographic and pathologic correlation. Pediatr Radiol. 2010 Sep; 40(9): 1562-5.

13. Lee EY. Intersttial lung disease in infants: new classification system, imaging technique, clinical presentation and imaging findings. Pediatr Radiol. 2013 Jan; 43(1): 3-13; quiz p.128-9.

14. Radman MR, Goldhoff $\mathrm{P}$, Jones $\mathrm{KD}$, et al. pulmonary interstitial glycogenosis: an unrecognized etiology of persistent pulmonary hypertension of the newborn in congenital heart disease? Pediatr Cardiol. 2013 Jun; 34(5): 1254-7.

15. King BA, Boyd JT, Kingma PS. Pulmonary maturational arrest and death in a patient with pulmonary interstitial glycogenosis. Pediatr Pulmonol. 2011 Nov; 46(11): 1142-5.

16. Alkhorayyef A, Ryerson L, Chan A, et al. pulmonary Interstitial glycogenosis associated with pulmonary hypertension and hypertrophic cardiomyopathy. Pediatr Cardiol. 2013 Feb; 34(2): 4626.

17. Still GG, Li S, Wilson M, et al. Persistent pulmonary hypertension without underlying cardiac disease as a presentation of pulmonary interstitial glycogenosis. Fetal Pediatr Pathol. 2018 Feb; 37(1): 22-26. 
18. Demirel N, Ochoa $\mathrm{R}$, Dishop $\mathrm{MK}$, et al. Respiratory distress in 2-month-old infant: Is the primary cause cardiac, pulmonary or both? Respir Med Case Rep. 2018 Jun 19; 25: 61-65.

19. Deutsch GH, Young LR. Lipofibroblast phenotype in pulmonary interstitial glycogenosis. Am J Respir Crit Care Med. 2016 Mar 15; 193 (6): 694-6.

20. Sanchez-de-Toledo J, Gonzalez-Peris S, Gran F, et al. Pulmonary Interstitial Glycogenosis: A Reversible Underlying Condition Associated With D-Transposition of the Great Arteries and Severe Persistent Pulmonary Hypertension. World J Pediatr Congenit Heart Surg. 2015 Jul; 6(3): 480-3.

21. Siomos AK, Mitchell MB, Fonseca BM. Successful surgical repair of a massive window duct in a 1-month old with aniridia and pulmonary interstitial glycogenosis. Cardiol Young. 2015 Mar; 25(3): 594-6.

22. Ehsan Z, Montgomery GS, Tiller C. Kisling J, et al. An infant with pulmonary interstitial glycogenosis: clinical improvement is associated with improvement in the pulmonary diffusion capacity. Pediatr Pulmonol. 2014 Mar; 49(3): E17-20.
23. Ross MK, Ellis LS, Bird LM, et al. Pulmonary interstitial glycogenosis in a patient ultimately diagnosed with Noonan syndrome. Pediatr Pulmonol. 2014 May; 49(5): 508-11.

24. Smets K, Van Daele S. Neonatal pulmonary interstitial glycogenosis in a patient with hunter syndrome. Eur J Pediatr. 2011 Aug; 170(8): 1083-4.

25. Smets k, Dhaene K, Schelstraete $P$, et al. Neonatal pulmonary interstitial glycogen accumulation disorder. Eur J Pediatr. 2004 Jul; 163(7): 408-9.

26. Lanfranchi M, Allbery SM, Wheelock L, et al. Pulmonary interstitial glycogenosis. Pediatr Radiol. 2010 Mar; 40(3): 361-5.

27. Onland W, Molenaar JJ, Leguit RJ, et al. Pulmonary interstitial glycogenosis in identical twins. Pediatr Pulmonol. 2005 Oct; 40(4): 362-6.

28. Perea L, Blinman T, Piccione J, et al. Bilateral congenital lobar emphysema: staged management. J Pediatr Surg. 2017 Sep; 52(9): 1442-1445. 